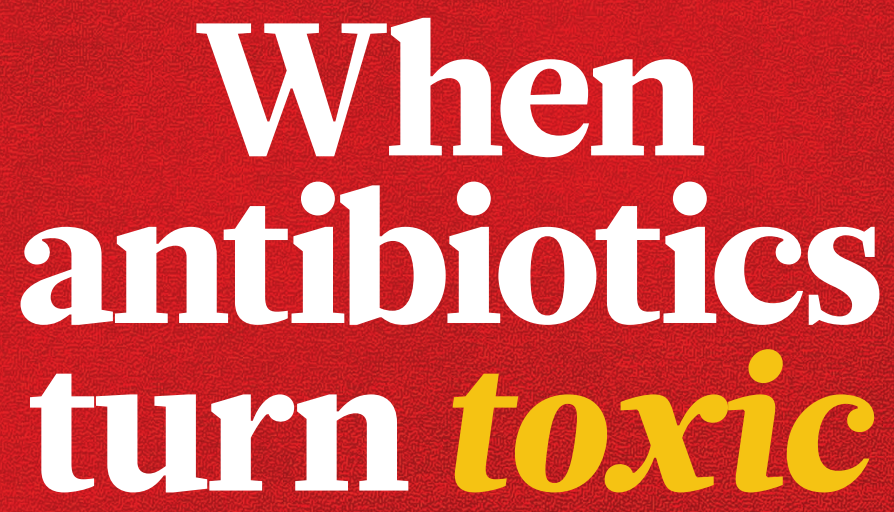

Researchers are asking why fluoroquinolone
drugs cause rare, disabling side effects.

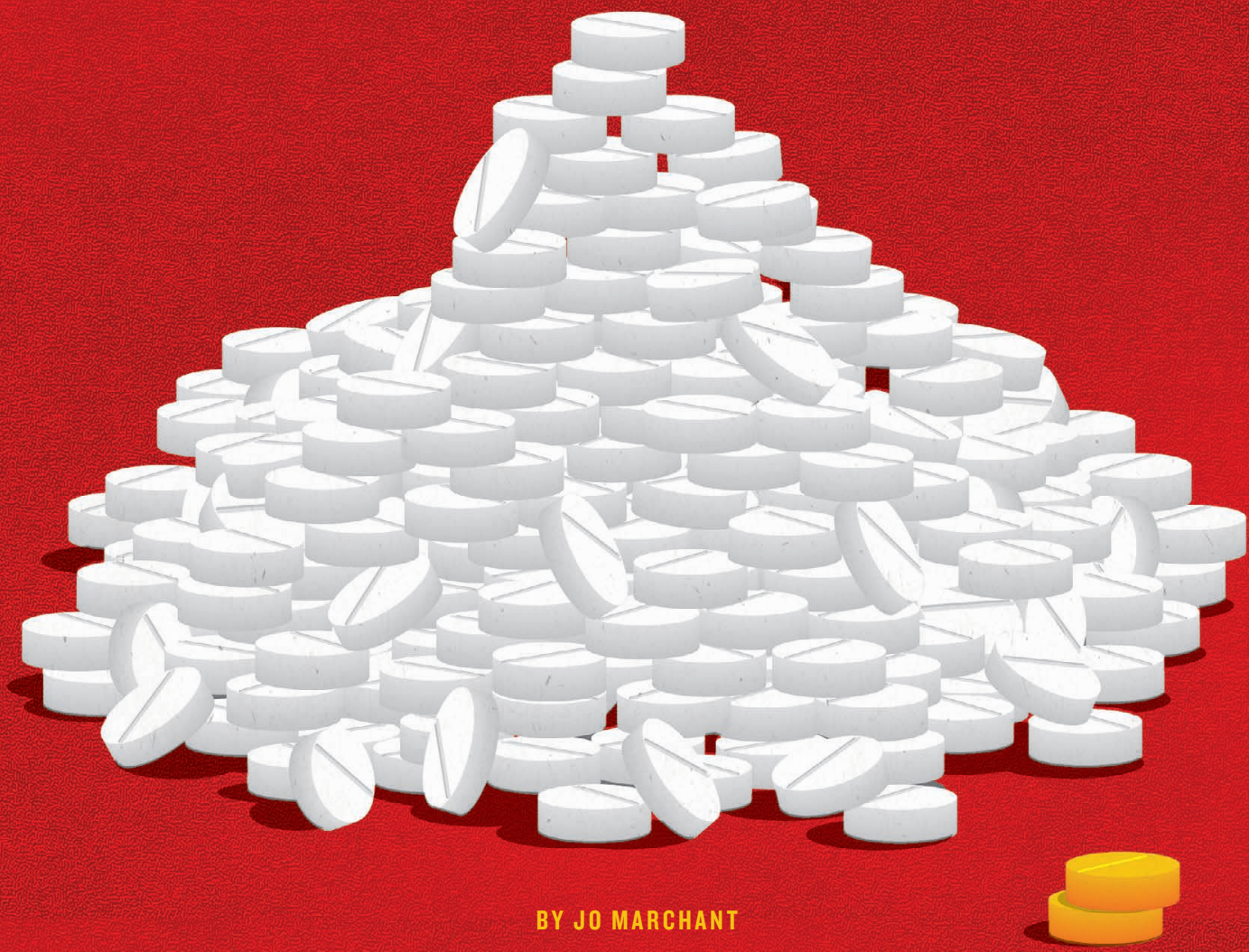

I n 2014, Miriam van Staveren went on holiday to the Canary Islands and caught an infection. Her ear and sinuses throbbed, so she went to see the resort doctor, who prescribed a six-day course of the popular antibiotic levofloxacin. Three weeks later, after she had returned home to Amsterdam, her Achilles tendons started to hurt, then her knees and shoulders. She developed shooting pains in her legs and feet, as well as fatigue and depression. "I got sicker and sicker," she says. "I was in pain all day." Previously an active tennis player and hiker, the 61-year-old physician could barely walk, and had to climb the stairs on all fours.

Since then, she has seen a variety of medical specialists. Some dismissed her symptoms as psychosomatic. Others suggested diagnoses of fibromyalgia or chronic fatigue syndrome. Van Staveren is in no doubt, however. She's convinced that the antibiotic poisoned her.

She's not alone. Levofloxacin is one of a class of drugs called fluoroquinolones, some of the world's most commonly prescribed antibiotics. In the United States in 2015, doctors doled out 32 million prescriptions for the drugs, making them the country's fourth-most popular class of antibiotic. But for a small percentage of people, fluoroquinolones have developed a bad reputation. On websites and Facebook groups with names such as Floxie Hope and My Quin Story, thousands of people who have fallen ill after fluoroquinolone treatment gather to share experiences. Many of them describe a devastating and progressive condition, encompassing symptoms ranging from psychiatric and sensory disturbances to problems with muscles, tendons and nerves that continue after people have stopped taking the drugs. They call it being 'floxed'.

For decades, regulatory agencies and the medical profession were sceptical that a brief course of antibiotics could have such a devastating, long-term impact. But after persistent campaigning by patient groups, attitudes began to change in 2008, when the US Food and Drug Administration (FDA) announced the first of what would be a series of strong alerts about the side effects of fluoroquinolone drugs, including tendon 
rupture and irreversible nerve damage. In 2016, the agency accepted the existence of a potentially permanent syndrome that it calls fluoroquinolone-associated disability (FQAD), and recommended that the drugs be reserved for serious infections. That move has triggered other regulatory agencies to reassess the antibiotics: Health Canada warned doctors of rare cases of persistent or disabling side effects in January 2017, and the European Medicines Agency (EMA) is expected to publish the results of a safety review this year, after a public hearing planned for June.

Fluoroquinolones are valuable antibiotics, and safe for most people. Yet they are so widely prescribed that their side effects might have harmed hundreds of thousands of people in the United States alone, say scientists who are working with patients to unpick FQAD's causes. Fluoroquinolone toxicity, they say, provides a compelling example of an emerging understanding that antibiotics don't just harm microbes they can severely damage human cells, too. Until recently, investigations into the side effects of antibiotics have focused on how the drugs disrupt the human microbiome, says James Collins, a medical engineer at the Massachusetts Institute of Technology in Cambridge. "Antibiotics are also disrupting our cells, and in pretty hefty ways," he says.

\section{THE DARK SIDE OF FLUOROQUINOLONES}

Quinolone antibiotics, first developed in the 1960 s, kill bacteria by blocking enzymes called class II topoisomerases, which normally untangle DNA during cell replication.

These enzymes usually cut DNA's double helix, pass another part of the strand through the gap, and then mend the cut. But quinolones bind to the enzymes, preventing them from mending their cuts. In the 1980 s, researchers added fluorine atoms to the quinolones' structures. This allowed the antibiotics to penetrate tissues throughout the body, including the central nervous system, and boosted their effectiveness against a broad range of bacterial infections.

Some FDA-approved fluoroquinolones were swiftly withdrawn from the market after severe adverse reactions and several deaths - trovafloxacin, withdrawn in 1999, damaged livers, for instance. But others became the drug of choice both for serious infections and for routine complaints, despite rare side effects. "These are heavily used drugs because they are very effective," says Joe Deweese, a biochemist who studies topoisomerases at Lipscomb University College of Pharmacy in Nashville, Tennessee. In the 1990s, ciprofloxacin (cipro) was given to US troops serving in the Persian Gulf as prophylaxis in case of exposure to anthrax spores. And in 2001, sales of cipro surged after a series of terrorist attacks involving anthrax; the US Centers for Disease Control and Prevention (CDC) recommended a 60-day course for anyone at risk of being exposed.

But by that point, some people had already flagged potential problems. In 1998, US journalist Stephen Fried (now at Columbia Journalism School in New York) published a book called Bitter Pills about his wife's severe and long-lasting neurological reaction to ofloxacin. It helped to trigger a wave of reports on websites such as the Quinolone Antibiotics Adverse Reaction Forum, which by 2001 hosted more than 5,000 posts. The late Jay Cohen, then a psychiatrist and medical researcher at the University of California, San Diego, contacted patients through the sites and published 45 case studies $^{1}$. Cohen warned that after taking fluoroquinolones, some people had developed serious problems in multiple organs. These effects came on rapidly and lasted for months or years.

Cohen's work was largely dismissed at the time because of his reliance on online forums. But complaints and patient petitions continued. From the 1980s to the end of 2015, the FDA received reports from more than 60,000 patients detailing hundreds of thousands of 'serious adverse events' associated with the 5 fluoroquinolones still on the market (most commonly tendon rupture, as well as neurological and psychiatric symptoms), including 6,575 reports of deaths. The FDA says that the reports of adverse events it receives - sent in by drug manufacturers, by doctors and directly by consumers - cannot be used to reach conclusions about the severity of problems associated with drugs. Still, the fluoroquinolones have attracted more complaints than other more widely used antibiotics. And only $1-10 \%$ of adverse events are estimated to be reported to the FDA, suggesting that fluoroquinolones might have harmed hundreds of thousands of people in the United States alone, says Charles Bennett, a haematologist at the University of South Carolina's College of Pharmacy in Columbia. Bennett is also director of the Southern Network on Adverse Reactions, a state-funded pharmaceutical-safety watchdog, which has been working with people affected by fluoroquinolones since 2010 .

In 2008, the FDA announced 'black box' warnings of tendon rupture among those given the antibiotics; in 2013, it added a risk of irreversible nerve damage. (Such warnings are placed inside a black box on drug labels, and call attention to serious or life-threatening risks.) As alerts mounted, patients launched lawsuits against manufacturers of the drugs, claiming they had not been adequately informed of risks. These cases have been variously won, lost or settled for undisclosed sums, and many are still in progress; manufacturers argue that they handled risks appropriately, and work with the FDA to update safety labels.

In November 2015, the FDA voted to recognize FQAD as a syndrome on the basis of 178 cases that the agency regarded as clear-cut: otherwise healthy people who took fluoroquinolones for minor ailments and then developed disabling and potentially irreversible conditions ${ }^{2}$. The FDA also noted a disturbing pattern: fluoroquinolones had a much higher percentage of disabilities among their serious-adverseevent reports than did other antibiotics.

\section{MITOCHONDRIAL DAMAGE}

Beatrice Golomb at the University of California, San Diego, has been working for a decade with people affected by fluoroquinolones, beginning with David Melvin, a police officer and keen cyclist who had to use a wheelchair after he was given levofloxacin for suspected epididymitis in 2007. Accumulating evidence, Golomb says, suggests that fluoroquinolones are damaging mitochondria, the power packs inside human cells that evolved from symbiotic, bacteria-like cells billions of years ago. This kind of harm can affect every cell in the body, explaining why a wide range of symptoms can appear and get worse over time.

Mitochondrial toxicity is a problem with many classes of drug, says Mike Murphy, who studies the biology of mitochondria at the University of Cambridge, UK. But because mitochondria retain some similarities to their bacterial ancestors, antibiotics can pose a particular threat to them. Researchers have shown, for example, that aminoglycoside antibiotics can cause deafness by damaging mitochondria in the hair cells of the ear ${ }^{3}$.

Isolated studies from the 1980s onwards have suggested that fluoroquinolones impair mitochondrial function, but a 2013 study ${ }^{4}$ by Collins and his colleagues is the most convincing, researchers say. They reported that antibiotics in several classes triggered oxidative stress - a build-up of reactive, oxygen-containing molecules - in mitochondria, inhibiting their function across a range of mammalian cells, as well as in mice. "We were surprised at how strong the effect was and how common the effect was across the different classes," Collins says. But "the largest effects were seen in the quinolones".

Pharmaceutical researchers had spotted the issue, too: in 2010, toxicologist Yvonne Will and her colleagues at Pfizer in Groton, Connecticut, reported an assay to detect mitochondrial damage early in drug development ${ }^{5}$. They found that some antibiotics affected mitochondria and others didn't. Every fluoroquinolone they tested damaged mitochondria in human liver cells - having what the researchers described as " $a$ strong effect" at therapeutic concentrations, although Will cautions that it isn't possible to extrapolate from that result to clinical outcomes.

But the potential for mitochondrial damage still isn't widely appreciated 
among antibiotics researchers and the medical community, Collins says. "I think people generally assume that antibiotics do not impact mammalian cells," he says. One problem is that there is still no reliable biomarker that researchers can use to test for mitochondrial damage in people, tying cell-line research to clinical experience. Nor is it known precisely how the fluoroquinolones are damaging human cells. A 2013 FDA review of the antibiotics' safety, for example, cited a 1996 study $^{6}$ reporting that cipro caused DNA breaks in mitochondria in a variety of mammalian cell lines. But Neil Osheroff, a biochemist at Vanderbilt University in Nashville, Tennessee, who studies fluoroquinolones, is doubtful about that result. He has done his own lab tests, and found that, at therapeutic concentrations, the fluoroquinolones prescribed by doctors have very little effect on human $\mathrm{DNA}^{7}$. Meanwhile, mitochondrial damage isn't the only theory in play: a 2015 study, done on human kidney cells ${ }^{8}$, reported that fluoroquinolones can bind to iron atoms from the active sites of several enzymes that modify DNA, leading to epigenetic changes that might be related to some of the drugs' side effects.

At a conference last September, Bennett reported preliminary data that might hint at why only some people develop serious side effects from fluoroquinolones. He took saliva samples from 24 people who reported neuropsychiatric side effects - such as memory loss, panic attacks and depression - and found that 13 of them (57\%) shared a gene variant usually seen in only $9 \%$ of the population. Bennett is not revealing the gene's identity because he has a patent application in process, but he says that it seems to be a site related to poor metabolism of the quinolones. Such a mutation might cause dangerously high levels of the drug to accumulate in cells, including in the brain. Bennett is now conducting a trial with 100 more participants to see if he can replicate the result. If so, that might lead to a genetic test to identify people who should not be given the drugs.

\section{LACK OF SUPPORT}

Most scientists asked by Nature about fluoroquinolones said that more research is needed to understand their side effects. Collins hopes to explore mitochondrial damage by antibiotics in other animal models. He and Murphy have also found, in lab studies ${ }^{4,9}$, that giving antioxidants alongside fluoroquinolones seems to mitigate the effects on mitochondria. Murphy is interested in trials to avoid mitochondrial toxicity in drugs; he owns shares in a company that aims to set some up. But such trials are difficult and expensive, particularly for drugs that are given in sometimes life-threatening situations, he says. Golomb is currently conducting an unfunded online survey to gather information on the experiences of thousands of patients. She hopes that it will lead to hypotheses about what might mitigate harms that could then be tested in clinical trials. But little support is available. That's typical for research on drug safety. Investigating medications that have been on the market for years isn't a priority for research agencies such as the US National Institutes of Health, says Bennett. Manufacturers don't have an incentive to fund post-market safety studies, particularly for off-patent drugs such as cipro and levofloxacin, where the vast majority of sales are from generics firms. "So there is really nobody to champion this work," says Bennett.

Another factor is scientists' reluctance to publish results that drug companies might find unfavourable. "There's a long history of adverse action against people who expose drug and chemical harms," says Golomb. She cites a list made by the pharmaceutical firm Merck of doctors who criticized the anti-inflammatory drug Vioxx (rofecoxib), which was withdrawn from the market over an increased risk of heart attack and stroke. According to internal e-mails read out in court in 2009 as part of a Vioxx class-action case in Australia, a list e-mailed among Merck employees contained doctors' names with the labels "neutralize", "neutralized" or "discredit" next to them. (Merck did not respond to Nature's request for comment.) Aggressive tactics are "a very big problem", says Bennett, who says he has been threatened by drug companies in the past.

For Osheroff, there are morepressing concerns about fluoroquinolones, such as how to combat the emergence of antibiotic-resistant infections and develop new antibiotics. But he and other scientists agree that doctors should not prescribe fluoroquinolones for relatively minor infections when they could use other drugs. The FDA's warnings on drug labels, however, have been slow to produce results. Prescriptions for the drugs did not fall between 2011 and 2015, according to the CDC (see 'Overprescribed antibiotics'). This suggests, says Bennett, that official alerts haven't been enough to get physicians to change their habits. The labels for fluoroquinolones alone have changed around 20 times in the past 4 or 5 years, he says. "It is almost impossible for doctors to keep up." Still, US prescriptions of fluoroquinolone antibiotics did drop by around 10\% in 2016, and totals for the first half of 2017 suggest that prescriptions fell again last year, according to unpublished figures given to Nature by IQVIA, a health-data firm in Durham, North Carolina.

Meanwhile, van Staveren, in Amsterdam, complains that despite her medical training, she still can't find a doctor who believes her. She is waiting for the EMA to make its recommendations, and hopes that it will follow the FDA in acknowledging FQAD, and warning about it. (The EMA declined to comment while the review is ongoing.) "I want doctors to be informed about the risks, no matter how rare or not they are," van Staveren says. "I want warnings all over and I want the warnings to be taken seriously."

Jo Marchant is a science journalist based in London.

1. Cohen, J. S. Ann. Pharmacother. 35, 1540-1547 (2001).

2. Tennyson, L. E. \& Averch, T. D. Urology Pract. 4, 383-387 (2016).

3. Gao, Z., Chen, Y. \& Guan, M.-X. J. Otol. 12, 1-8 (2017).

4. Kalghatgi, S. et al. Sci. Transl. Med. 5, 192 ra85 (2013)

5. Nadanaciva, S. et al. Biomol. Screen. 15, 937-948 (2010).

6. Lawrence, J. W. et al. Mol. Pharmacol. 50, 1178-1188 (1996)

7. Aldred, K. J. et al. ACS Chem. Biol. 8, 2660-2668 (2013).

8. Badal, S. et al. J. Biol. Chem. 290, 22287-22297 (2015).

9. Lowes, D. A. et al. Free Radical Res. 43, 323-328 (2009). 